\title{
Long Term Maintenance Therapy with VEGF Inhibitor with Remission of the Disease for Seven Years in Patient with Metastatic Rectal Cancer
}

\author{
Mesti Tanja, Reberšek Martina and Ocvirk Janja* \\ Division of Medical Oncology, Institute of Oncology Ljubljana, Slovenia
}

Submission: March 12, 2019; Published: March 22, 2019

*Corresponding author: Janja Ocvirk, M.D., Ph.D., Institute of Oncology Ljubljana, Zaloška 2, Ljubljana, Slovenia

\begin{abstract}
We report a case of a 65-year-old female with metastatic rectal adenocarcinoma which was on maintenance therapy with bevacizumab from September 2007 to September 2014. For seven years she was in remission. At that time except for the hoarseness that developed after three years of maintenance therapy with bevacizumab, no additional changes were noted. Bevacizumab is a monoclonal antibody, a VEGF inhibitor. Neutralizing the biological activity of VEGF regresses the vascularisation of tumours, normalises remaining tumour vasculature, and inhibits the formation of new tumour vasculature, thereby inhibiting tumour growth. The use of maintenance therapy with bevacizumab in patients with metastatic colorectal cancer is widely accepted, but the duration of maintenance is not yet defined. The side effects of bevacizumab as maintenance therapy remains to be answered. The individual treatment approach is the best way of treating the patient, and in the lack of definite data of the biology of the individual metastatic carcinoma, the multidisciplinary approach and the experienced specialists are essential.
\end{abstract}

Keywords: Maintenance therapy with bevacizumab; Metastatic colorectal cancer; Late side effects of VEGF inhibitors

\section{Introduction}

Bevacizumab is a monoclonal antibody, which binds to VEGF, the key driver of neovascularization, and thereby inhibits the binding of VEGF to its receptors, VEGFR-1 and VEGFR-2, on the surface of endothelial cells. Neutralising the biological activity of VEGF regresses the vascularisation of tumours, normalises remaining tumour vasculature, and inhibits the formation of new tumour vasculature, thereby inhibiting tumour growth [1].

Anti-VEGF therapy, including bevacizumab, acts by binding to VEGF and preventing its cellular effects. However, this linear interaction represents only a partial view of the pathobiology of the disease and treatment processes. Consequently, the classical concept of linear interactions is being replaced by the concept of networks of interactions, emphasizing the importance of interactions between different components of a biologic system $[2]$.

Bevacizumab in combination with different types of chemotherapy is registered for the treatment of patients with metastatic carcinoma of the colon or rectum, metastatic breast cancer, advanced and/or metastatic renal cancer, unresectable advanced, metastatic or recurrent non-small cell lung cancer with other than predominantly squamous cell histology, advanced (International Federation of Gynecology and Obstetrics (FIGO) stages III B, III C and IV) epithelial ovarian, fallopian tube or primary peritoneal cancer and persistent, recurrent, or metastatic carcinoma of the cervix. Bevacizumab demonstrated significant clinical activity in phase II studies using bevacizumab as a single agent or in combination with chemotherapy agents such as irinotecan for patients with grade 3 and grade 4 malignant gliomas, and has been already approved by FDA, but not by EMA [3-5].

We reporta case of a patient, with metastatic adenocarcinoma of the rectum, on maintenance therapy with bevacizumab from September 2007 till September 2014, being in disease remission for seven years, and only with dysphonia as side effect. We want to rise attention on two questions: duration of the maintenance therapy with bevacizumab in mCRC and its late side effects.

\section{Case Report}

The 49-year-old female was diagnosed with metastatic rectal adenocarcinoma with liver metastases in April 1995 and underwent the resection of the primary tumor and liver metastases, stage T3N2M1, after preoperative chemoradiotherapy with 5-Fluorouracil (5-FU) and Cisplatin. 
Year 2000 at progression, the second resection of the solitary liver metastasis (mets) was performed. In May 2004 the mets in scl left near art. Carotis communis was diagnosed and she received radical RT (45Gy) as the disease was inoperabile, afterwards stagnation of the disease was achieved. In October 2006 she presented with Horner Sy, because of the inoperabile disease progression in the left scl and two mets in the VI and VII part of the liver (Figure 1). From November 2006 till February 2007 she received four cycles of Capecitabin and Irinotecan (XELIRI) in combination with bevacizumab. The Horner Sy disappeared. The control CT of the thorax and abdomen has showed partial response of the disease in the left scl and in the liver, so the metastasectomy of the liver mets followed in March 2007. She continued with two additional cycles of XELIRI + bevacizumab till May 2007. At that time the beta blocator and thyroxin substitution was introduced as regular therapy by the cardiologyst because of tachicardia as late side effect of received RT of the left scl. In June 2007 asymptomatic pulmonary microembolisms were diagnosed and she started with therapeutic doses of low weight heparin and systemic therapy was discontinued. After one month the condition has normalized, the hearth function remained normal (EF LV $55 \%$ ) and as the control CT of the thorax and abdomen showed further regression of the disease in the left scl, she received two additional cycles of XELIRI combined with bevacizumab. Afterwards she continued with maintenance monotherapy with bevacizumab.

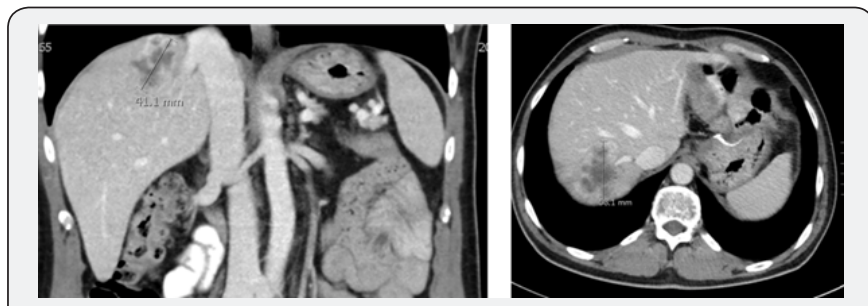

Figure 1: CT Imaging - Unresectable dissemination of the disease in the liver, October 2006.

For seven years, from September 2007 till September 2014 she undertook maintenance therapy with bevacizumab $7,5 \mathrm{mg} /$ $\mathrm{kg}$ ) every three weeks with the cancer disease in remission (Figure 2). The follow up was performed by CT of the neck, thorax and abdomen every 6 months.

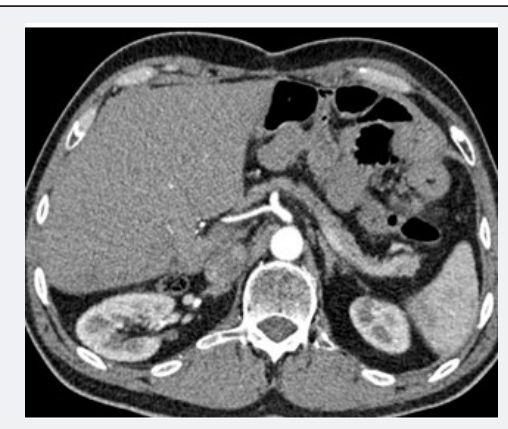

Figure 2: MRI - CR in the liver. February 2010.
After three years of maintenance therapy she presented with hoarseness. Esophago-gastro-duodenoscopy (EGDS) showed no signs of reflux esophagytis or inflammatory changes. The fiberoptic laryngeal examination showed abnormally whitish, atrophic vocal folds with abnormale mobility of the left vocal cord, as a result of paresis of n.reccurens and laryngeus superior. The etiology of the paresis is still unknown. CT of the neck, thorax and abdomen in January 2010 showed unchanged situation as remission remained.

The tumour tissue from the resected primary carcinoma was assessed for biomarkers status in July 2013 and showed KRAS mutation present on codon 13 .

In September 2014 the progression of the disease in the lymph nodes of the neck and in the abdomen as in the liver was diagnosed and the treatment with bevacizumab was discontinued.

\section{Discussion}

In the Bevacizumab metastatic colorectal cancer (mCRC) pivotal studies called for treatment to be continued until disease progression (PD) even if the corresponding chemotherapy was modified or discontinued. The pivotal studies include, AVF2107g (IFL irinotecan/5fluorouracil/leucovorin + Bevacizumab for the first line), E3200 (for the second line FOLFOX4 5fluorouracil/ leucovorin/oxaliplatin + Bevacizumab in Bevacizumab naive patients), and ML18147 (first through second line, FP fluoropyrimidine + oxaliplatin or irinotecan-based chemotherapy + Bevacizumab). The Bevacizumab was continued until PD in $77 \%, 88 \%$ and $72 \%$ of patients respectively. Bevacizumab treated patients experienced significant improvements in overall survival (OS) and progression free survival (PFS) compared with patients in the control arm [6-8].

In the multinational Phase 3 study N016966 that evaluated the safety and efficacy of XELOX capecitabin/oxaliplatin + Bevacizumab vs FOLFOX4 + Bevacizumab in first line mCRC patients $(\mathrm{N}=1400)$, patients were randomized to receive FOLFOX4 + Bevacizumab, FOLFOX4 + placebo, XELOX + Bevacizumab, or XELOX + placebo for 48 weeks or until PD or death. The PFS significantly improved in the Bevacizumab + XELOX arm compared with the chemotherapy alone arm (9.3 months vs 7.4 months, hazard ratio HR 0.77, p=0.0026). No significant difference was observed in the Bevacizumab + chemotherapy arm vs chemotherapy alone arm in median OS (21.3 months vs 19.9 months, $\mathrm{p}=0.077$ ) and response rate RR ( $47 \%$ vs $49 \%, p=0.31$ ). Median time to treatment failure was 6 months in the chemotherapy alone arm and 6.9 months in the Bevacizumab + chemotherapy arm $(p=0.003)$ [9].

In the open label randomized Phase 3 CAIRO3 study, previously untreated mCRC patients who achieved stable disease SD or better after 6 cycles of induction therapy with CAPOX-B capecitabin/oxaliplatin/bevacizumab $(n=558)$ were randomized to either maintenance therapy with Bevacizumab 
$(7.5 \mathrm{mg} / \mathrm{kg}$ every three weeks) + capecitabine $(625 \mathrm{mg} / \mathrm{m} 2$ twice daily) or observation only. Upon PD, patients (regardless of the initial treatment arm) were reintroduced to CAPOX-B and received continued therapy until $\mathrm{PD}$, death or an unacceptable adverse event AE. The primary end point of second PFS was significantly improved in Bevacizumab/capecitabine arm compared with observation only maintenance therapy (11.7 months vs 8.5 months, stratified HR 0.67, p<0.0001). This is the only study that compared maintenance Chemotherapy + Bevacizumab vs Bevacizumab and showed noninferiority of maintenance monotherapy Bevacizumab vs combination therapy, so maintenance therapy with single agent Bevacizumab may be an appropriate option following induction XELOX + Bevacizumab mCRC patients [10].

In the multicentre randomized Phase 3 MACRO study, a total of 480 patients with previously untreated mCRC were randomized to receive Bevacizumab $(7.5 \mathrm{mg} / \mathrm{kg}$ every 3 weeks) + XELOX for 6 cycles followed by maintenance therapy every 3 weeks with continued Bevacizumab + XELOX $(n=239)$ or single agent Bevacizumab (n=241) until PD or unacceptable AE.8 The study was designed as a non-inferiority study with a prespecified non-inferiority PFS HR limit of 1.32. The non-inferiority of the single agent Bevacizumab could not be confirmed when compared with Bevacizumab + XELOX maintenance therapy (median PFS 9.7 months vs 10.4 months, stratified HR 1.1, $\mathrm{p}=0.38)[11]$.

The DREAM study was multicentre Phase 3 study that compared maintenance therapy with Bevacizumab $(7.5 \mathrm{mg} / \mathrm{kg}$ every 3 weeks $)+$ erlotinib (150 mg/day) $(\mathrm{n}=224)$ with single agent Bevacizumab $(n=228)$ after induction therapy with investigators choice of Bevacizumab (5 mg/kg every 2 weeks) + mFOLFOX7 modified FOLFOX $(\mathrm{n}=429)$, biweekly mXELOX modified XELOX ( $\mathrm{n}=204)$, or FOLFIRI 5fluorouracil/leucovorin/ irinotecan $(n=67)$ in 700 previously untreated patients with mCRC. The primary end point of maintenance PFS was significantly improved Bevacizumab + erlotinib compared with single agent Bevacizumab maintenance therapy (5.9 moths vs 4.9 months, HR 0.77, p=0.012) [12].

The Phase 3 PRODIGY 9 study evaluated no maintenance therapy (observation only) compared with Bevacizumab maintenance during CFI chemotherapy free interval following induction treatment in patients with mCRC. Patients were randomized to 12 cycles of induction therapy with Bevacizumab + FOLFIRI arm followed by CFI until PD. Chemotherapy was restarted at PD for 8 cycles followed again by CFI, this method was continued. The primary end point of tumour control duration was not significantly different between single agent Bevacizumab and observation only maintenance therapy (15.08 months vs 14.98 months, HR 1.09, p=0.43) during CFI before PD and chemotherapy re-initiation [13].

As result of the CAIRO3 study, the maintenance therapy with combination of capecitabin and bevacizumab is nowadays standard of treatment [10]. In the time when our patient was treated there were not enough data to support this, so the maintenance therapy with bevacizumab only was applied.

Bevacizumab is associated with a broad spectrum of adverse effects and may be fatal in a small number of cases. Across Phase 3 studies, adverse events reported as very common and common with Bevacizumab included gastrointestinal perforation, thromboembolic events, hypertension, neutropenia, febrile neutropenia, proteinuria, Bleeding, leukopenia, diarrhea, vomiting, nausea, anemia, thrombocytopenia, cardiac disorders, neurotoxicity, hand-foot syndrome, fatigue, abdominal pain, fever, neuropathy, rash, ileus, dehydration, headache, dyspnea, mucositis [6-13].

Some of these adverse effects are described when bevacizumab is used as combination wich chemotherapeutic agents, like the tromboembolic events, as was the case with our patients. The decision to continue with the combination of chemotherapy and bevacizumab was made on the basis of the patients benefit and preferences, but not before the good hearth and pulmonary circulation function was assessed. Many others side effects that are unique and not typically observed with chemotherapy, so it is questionable which of these are associated with prolonged bevacizumab maintenance therapy.

In our patient hoarseness occurred as the only side effect after three years of maintenance therapy with bevacizumab, without signs of reflux esophagytis or inflamatory changes and with atrophia of the vocal folds with abnormale mobility of the left vocal cord, as a result of paresis of n.reccurens and laryngeus superior. The patient has been treated with radiotherapy in the neck area, which could be the possible reason for dysphonia. It is also possible that Bevacizumab has made additional changes and contributed to the development of the dysphonia. Still leaves unclear the etiology of the dysphonia in our patient.

Hartl et al. [14] in 2010 first reported a case of dysphonia with laryngeal necrosis induced after three weeks of treatment with bevacizumab [14]. Afterwards there has been other similar cases reported in patients in the early setting of bevacizumab administration $[15,16]$. Temporary interruption of maintenance therapy resolves the complication. The decision of restarting and continuation of maintenance therapy with Bevacizumab must be made due to the potential benefits, quality of life and patients' preferences [14-16].

In almost all studies analyzing the treatment with antiangiogenic inhibitors (bevacizumab, aflibercept, sunitinib, sorafenib, pazopanib, axitinib and regorafenib) has been found and reported the certain degree of dysphonia. Voice changes has especially been noticed with the use of axitinib, aflibercept and regorafenib. The angiogenesis inhibition possibly plays a role by altering the larynx in some way and modifying vocal fold vibrations, leading to dysphonia [17]. The physiologic importance of VEGF signalling in the larynx remains incompletely defined. 
VEGF may either promote, inhibit, or have no effect on laryngeal wound healing. Laryngeal inflammation due to either reflux or Reinke's oedema has been positively associated with VEGF upregulation, though this would predict an antilaryngitis effect of VEGF signaling inhibition. Neurotoxicity or vasculopathic laryngeal nerve damage, may also be a possible mechanism of toxicity of the VEGF inhibitors [14-17].

Dysphonia is now reported as common side effect of Bevacizumab, in the time of our patient treatment this was still unknown [18].

\section{Conclusion}

Many data show that continued suppression of the VEGF pathway may be important to maximize the clinical benefit from bevacizumab in mCRC. Although there are several studies that provide data for maintenance treatment with Bevacizumab + Chemotherapy, the question whether to continue with bevacizumab as monotherapy after evidence of complete response and the duration of treatment remains unclear. Only one study compared maintenance Chemotherapy + Bevacizumab vs Bevacizumab as monotherapy and as presented previously showed noninferiority of bevacizumab versus combination therapy [10], so maintenance therapy with single-agent bevacizumab may be an appropriate option following induction XELOX plus bevacizumab in $\mathrm{mCRC}$ patients.

The second point of duration of maintenance therapy with bevacizumab especially after complete response has no response for now and only can be related to the toxicity profile of the drug and the risk of complications, especially thromboembolic or hemorrhagic. We can also consider the economical aspect, which assumes even greater importance in view of the general economic situation and finally the patient wishes, and quality of life must be taken into consideration.

Hoarseness and general voice disorders are not lifethreatening side effects, but they could considerably affect quality of life. Clinicians should not underestimate this type of toxicity. The early recognition of VEGF-dependent vocal pathology is important. If symptoms are severe and/or progressive, immediate discontinuation of the anti-angiogenic drug may be prudent, as the possibility of a persistent deficit and possible permanent malfunctioning seems real. Whether drugs such as steroids or antiplatelet agents may be helpful in prevention or treatment is not yet clear and could be studied in future patients. The question of late side effects of Bevacizumab such as reflux laryngitis and its mechanism remain open. While waiting for answers the best approach is to adjust the treatment to the patient individually.

\section{References}

1. Ferrara N, Hillan KJ, Novotny W (2005) Bevacizumab (Avastin), a humanized anti-VEGF monoclonal antibody for cancer therapy, Biochem Biophys Res Commun 333(2): 328-335.
2. Loscalzo J, Kohane I, Barabasi AL (2007) Human disease classification in the postgenomic era: a complex systems approach to human pathobiology. Mol Syst Biol 3: 124.

3. Kreisl TN, Kim L, Moore K, Duic P, Royce C, et al. (2009) Phase II trial of single-agent bevacizumab followed by bevacizumab plus irinotecan at tumor progression in recurrent glioblastoma. J Clin Oncol 27(5): 740745 .

4. Friedman HS, Prados MD, Wen PY, Mikkelsen T, Schiff D, et al (2009) Bevacizumab alone and in combination with irinotecan in recurrent glioblastoma. J Clin Oncol 27(28): 4733-4740.

5. Cloughesy T, Vredenburgh JJ, Day B, Das A, Friedman HS, et al. (2010) Updated safety and survival of patients with relapsed glioblastoma treated with bevacizumab in the BRAIN study (abstract \#2008). J Clin Oncol 28: 181s.

6. Hurwitz F, Fehrenbacher L, Novotny W, Cartwright T, Hainsworth J et al. (2004) Bevacizumab plus itinotecan, fluorouracil, and leucovorin for metastatic colorectal cancer. N Engl J Med 350(23): 2335-2342.

7. Giantonio BJ, Catalano PJ, Meropol NJ, O’Dwyer PJ, Mitchell EP, et al. (2007) Bevacizumab in combination with oxaliplatin, fluorouracil and leucovorin (FOLFOX4) for previously treated metastatic colorectal cancer: results from Eastern Cooperative Oncology Group study E3200. J Clin Oncol 25(12): 1539-1544.

8. Bennouna J, Sastre J, Arnold D, Osterlund P, Greil R et al. (2013) Continuation of bevacizumab after first progression in metastatic colorectal cancer (ML18147): a randomized Phase 3 trial. Lancet Oncol 14(1): 29-37.

9. Saltz LB, Clarke S, Díaz-Rubio E, Scheithauer W, Figer A, et al. (2008) Bevacizumab in combination with oxaliplatin-based chemotherapy as first-line therapy in metastatic colorectal cancer: a randomized phase III study. J Clin Oncol 26(12): 2013-2019.

10. Simkens LHJ, van Tinteren H, May A, ten Tije AJ, Creemers GJM, et al (2015) Maintenance treatment with capecitabine and bevacizumab in metastatic colorectal cancer (CAIRO3): a phase 3 randomised controlled trial of the Dutch Colorectal Cancer Group. The Lancet 385(9980): 1843-1852.

11. Díaz-Rubio E, Gómez-España A, Massutí B, Sastre J, Abad A, et al. (2012) First-line XELOX plus bevacizumab followed by XELOX plus bevacizumab or single-agent bevacizumab as maintenance therapy in patients with metastatic colorectal cancer: the phase III MACRO TTD study. Oncologist 17(1):15-25.

12. Tournigand C, Scheithauer W, Samson B, Lledo G, Viret F, et al. (2013) Induction treatment in first-line with chemotherapy + bevacizumab (bev) in metastatic colorectal cancer: Results from the GERCORDREAM phase III study. Presented at the 2013 Gastrointestinal Cancers Symposium in San Francisco, CA; January 24-26, ASCO GI Poster \#457.

13. Aparicio T, Bennouna J, Le Malicot K, Ghiringhelli F, Boige V et al. Final results of PRODIGE 9, a randomized phase III comparing no treatment to bevacizumab maintenance during chemotherapy-free intervals in metastatic colorectal cancer. Presented at the 2016 ASCO Annual Meeting in Chicago, IL; June 3-7, ASCO Abstract \#3531.

14. Hartl DM, Ferte C, Loriot Y, Roca CG, Bahleda R, et al. (2010) Dysphonia induced by vascular endothelium growth factor/vascular endothelium growth factor receptor inhibitors. Invest New Drugs 28(6): 884-886.

15. Caruso AM, Meyer TK, Allen CT (2014) Hoarseness after metastatic colon cancer treatment. JAMA Otolaryngol Head Neck Surg 140(9): 881-882.

16. Carter CA, Caroen SZ, Oronsky AL, Oronsky BT (2015) Dysphonia after Bevacizumab Rechallenge: A Case Report. Case Rep Oncol 8(3): 423425 . 
17. Saavedra E, Hollebecque A, Soria JC, Hartl DM (2014) Dysphonia induced by anti-angiogenic compounds. Invest New Drugs 32(4): 774 782
18. http://www.ema.europa.eu/docs/en_GB/document_library/EPAR_ Product_Information/human/000582/WC500029271.pdf

\section{Your next submission with Juniper Publishers will reach you the below assets}

- Quality Editorial service

- Swift Peer Review

- Reprints availability

- E-prints Service

- Manuscript Podcast for convenient understanding

- Global attainment for your research

- Manuscript accessibility in different formats

( Pdf, E-pub, Full Text, Audio)

- Unceasing customer service

Track the below URL for one-step submission https://juniperpublishers.com/online-submission.php 